\title{
Concept of Synthetic Lethality in Cancer
}

Targets that are present in tumor cells but absent in normal cells are the ideal or preferable cancer drug targets. Such cancer targets are defined as synthetic lethal interactions or as cancer-specific vulnerabilities with cancer-specific genetic lesions. The quest for synthetic lethal interactions concentrates on proteins that elude pharmacological inhibition but are frequently mutated. ${ }^{1}$

Calvin Bridges, in 1922, first described the phenomenon of synthetic lethality and noticed that in Drosophila melanogaster combinations of mutations confer lethality. ${ }^{2}$ The term 'synthetic lethality' was coined by Theodore Dobzhansky in 1946 to describe a similar type of genetic interaction in wild-type population of Drosophila. ${ }^{3}$

The term synthetic lethality refers to the interaction between two genetic components. Synthetic lethality also applies to cases in which the combination of a mutation and the action of a chemical compound causes lethality, whereas the mutation or compound alone is nonlethal but act synergistically. ${ }^{4}$

Exploiting synthetic lethal interactions with cancer-associated mutations are the recent efforts in the design of cancer therapy. To reduce off-target effects of chemotherapies and chemopreventive drugs, a synthetic lethal approach to cancer therapy is currently being explored.

Mutated oncogenes with increased activity are inhibited by a vast majority of targeted anticancer drugs. Yet many tumors do not contain such actionable aberrations, such as those harboring loss-of-function mutations. The concept of targeting synthetic lethal systems in cancer cells has provided a pathway to exploit more of the epigenetic and genetic changes acquired during carcinogenesis. ${ }^{5}$

Synthetic lethal analysis can be used to understand mechanisms of known chemotherapeutic drugs by identifying genes whose function is necessary for drug therapy. Mutations in BRCA1 and BRCA2 predispose individuals to breast and ovarian cancer. ${ }^{6}$ PARP1 is an enzyme involved in repairing single-stranded DNA breaks, and the inhibition of this enzyme is selectively lethal to such tumors as cancer cells accumulate DNA which cannot be repaired. Synthetic lethality is also used for screening purposes of gene libraries to detect drugs that specifically inhibit cancer cells. In a recent study, a compound of 3200 screened molecules was a synthetic lethal inhibitor of pancreatic cancer, such findings suggest a potential mode of treatment of pancreatic cancer. ${ }^{7}$

New synthetic lethal partners are created in cancer cells and are marked by errors in DNA repair, uncontrolled transcription and genetic instability. A cancer-related mutation can sensitize cancer cells to chemotherapy that target its synthetic lethal partner, since drugs act on a target-specific gene product which resembles the phenotype caused by a mutation. ${ }^{6}$

Synthetic lethality should be used to avoid off-target side effects of chemotherapeutics by targeting synthetic lethal partners of mutations in cancer cells which are nontoxic to normal cells.

\section{REFERENCES}

1. Kuiken HJ, Beijersbergen RL. Exploration of synthetic lethal interactions as cancer drug targets. Future Oncol 2010 Nov;6(11):1789-1802.

2. Nijman SB. Synthetic lethality: general principles, utility and detection using genetic screens in human cells. FEBS Lett 2011 Jan 3;585(1):1-6.

3. Ferrari E, Lucca C, Foiani M. A lethal combination for cancer cells: synthetic lethality screenings for drug discovery. Eur J Cancer 2010 Nov;46(16):2889-2895.

4. Hartwell LH, Szankasi P, Roberts CJ, Murray AW, Friend SH. Integrating genetic approaches into the discovery of anticancer drugs. Science 1997 Nov 7;278(5340):1064-1068.

5. Fece de la Cruz F, Gapp BV, Nijman SM. Synthetic lethal vulnerabilities of cancer. Annu Rev Pharmacol Toxicol 2015;55:513-531.

6. Farmer H, McCabe N, Lord CJ, Tutt AN, Johnson DA, Richardson TB, Santarosa M, Dillon KJ, Hickson I, Knights C, et al. Targeting the DNA repair defect in BRCA mutant cells as a therapeutic strategy. Nature 2005 Apr 14;434(7035):917-921.

7. Ji Z, Mei FC, Lory PL, Gilbertson SR, Chen Y, Cheng X. Chemical genetic screening of KRAS-based synthetic lethal inhibitors for pancreatic cancer. Front Biosci (Landmark Ed) 2009 Jan 1;14:2904-2910.

Shankargouda Patil

Associate Professor

College of Dentistry, Jazan University, Jazan, Saudi Arabia

Dominic Augustine

Assistant Professor

Department of Oral and Maxillofacial Pathology

Faculty of Dental Sciences, MS Ramaiah University of Applied Sciences MSR Nagar, Bengaluru, Karnataka, India

Roopa S Rao

Professor and Head

Department of Oral and Maxillofacial Pathology

Faculty of Dental Sciences, MS Ramaiah University of Applied Sciences MSR Nagar, Bengaluru, Karnataka, India 